\title{
Cryptotomography: Reconstructing 3D Fourier Intensities from Randomly Oriented Single-Shot Diffraction Patterns
}

\author{
N. D. Loh, ${ }^{1,11}$ M. J. Bogan, ${ }^{2}$ V. Elser, ${ }^{1}$ A. Barty, ${ }^{3}$ S. Boutet, ${ }^{2}$ S. Bajt, ${ }^{4}$ J. Hajdu, ${ }^{5}$ T. Ekeberg, ${ }^{5}$ F. R. N. C. Maia, ${ }^{5}$ J. Schulz, ${ }^{3}$ \\ M. M. Seibert, ${ }^{5}$ B. Iwan, ${ }^{5}$ N. Timneanu, ${ }^{5}$ S. Marchesini, ${ }^{6}$ I. Schlichting, ${ }^{7,8}$ R. L. Shoeman, ${ }^{7,8}$ L. Lomb, ${ }^{7,8}$ M. Frank, ${ }^{9}$ \\ M. Liang, ${ }^{3}$ and H. N. Chapman ${ }^{3,10}$ \\ ${ }^{1}$ Laboratory of Atomic and Solid State Physics Cornell University, Ithaca, New York 14853-2501, USA \\ ${ }^{2}$ SLAC National Accelerator Laboratory, 2575 Sand Hill Road, Menlo Park, California 94025, USA \\ ${ }^{3}$ Center for Free-Electron Laser Science, DESY, Notkestrasse 85, Hamburg 22607, Germany \\ ${ }^{4}$ Photon Science, DESY, Notkestrasse 85, Hamburg 22607, Germany \\ ${ }^{5}$ Laboratory of Molecular Biophysics, Department of Cell and Molecular Biology, Uppsala University, \\ Husargatan 3, Box 596, SE-75124 Uppsala, Sweden \\ ${ }^{6}$ Lawrence Berkeley National Laboratory, 1 Cyclotron Road, Berkeley, California 94720, USA \\ ${ }^{7}$ Max Planck Institute for Medical Research, Jahnstraße 29, 69120 Heidelberg, Germany \\ ${ }^{8}$ Max Planck Advanced Study Group, Center for Free-Electron Laser Science, DESY, Notkestrasse 85, Hamburg 22607, Germany \\ ${ }^{9}$ Lawrence Livermore National Laboratory, 7000 East Avenue, Livermore, California 94550, USA \\ ${ }^{10}$ University of Hamburg, Luruper Chaussee 149, Hamburg 22761, Germany \\ ${ }^{11}$ Cornell High Energy Synchrotron Source (CHESS), Cornell University, Ithaca, New York 14853-2501, USA
}

(Received 3 March 2010; published 2 June 2010; corrected 9 June 2010)

We reconstructed the 3D Fourier intensity distribution of monodisperse prolate nanoparticles using single-shot 2D coherent diffraction patterns collected at DESY's FLASH facility when a bright, coherent, ultrafast x-ray pulse intercepted individual particles of random, unmeasured orientations. This first experimental demonstration of cryptotomography extended the expansion-maximization-compression framework to accommodate unmeasured fluctuations in photon fluence and loss of data due to saturation or background scatter. This work is an important step towards realizing single-shot diffraction imaging of single biomolecules.

DOI: 10.1103/PhysRevLett.104.225501

PACS numbers: 61.05.cf, 41.60.Cr, 42.30.Rx, 42.55.Vc

Single-shot coherent diffraction imaging (CDI) is a developing technique aimed at determining the structure of very small biomolecules, such as proteins and viruses, not easily imaged through more established methods. In one scheme of single-shot CDI a serial stream of particles is injected into a pulse train of highly coherent and energetic $\mathrm{x}$-ray free-electron laser (FEL) radiation [1]. Photons from a single FEL pulse are diffracted when they encounter a particle. Although the FEL pulse destroys this particle, its structure is recorded in the diffraction data if the pulse is sufficiently short $[2,3]$.

As it is difficult to manipulate or determine the orientations of very small particles, they are currently injected into the FEL radiation at random, unmeasured orientations. Nevertheless, sufficiently many 2D diffraction patterns from an ensemble of identical albeit randomly oriented particles can in principle overdetermine the particle's band-limited 3D Fourier intensities. Earlier numerical simulations also show that the particle's 3D intensities can be recovered even if the random 2D diffraction patterns are remarkably noisy [4-6].

This Letter demonstrates the experimental feasibility of single-shot CDI with noisy 2D diffraction patterns from randomly oriented identical particles, which we coin cryptotomography. In this first exercise in cryptotomography, we reconstruct the 3D intensities of a relatively large and simple iron oxide nanoparticle. These nearly monodisperse particles are approximately solids of revolution with principal radii 25 and $100 \mathrm{~nm}$ [scanning electron microscope (SEM) measurements shown in Fig. 1].

Our primary objective is not to study these simple nanoparticles in any greater detail than what is already available via SEM, but to show that cryptotomography, despite its random and noisy data, is experimentally viable through the union of experimental and theoretical innovations. We do so in the style of previous papers in diffraction imaging, which demonstrated novel techniques with simple test subjects [3,7].

We performed our experiment at DESY's FLASH facility with each FEL pulse train comprising 100 pulses $(7 \mathrm{~nm}$ radiation; $30 \pm 10 \mu \mathrm{m}$ beam focus, extrapolated from [8]) separated by $10 \mu \mathrm{s}$, repeated at 5 pulse trains per second. Each detector exposure was $1 \mathrm{~s}$ long. Additional details on experimental parameters and data collection were similar to [1] and $[9,10]$, respectively, except we used a nebulizer instead to aerosolize the nanoparticles, which were then directed into the FEL radiation using an aerodynamic lens stack [11].

Our experiment imposed two considerable challenges. First, the FEL pulse fluence fluctuated due to electron bunch dynamics in the lasing process [12]. Second, there was considerable diffuse background scattering (Fig. 1) 


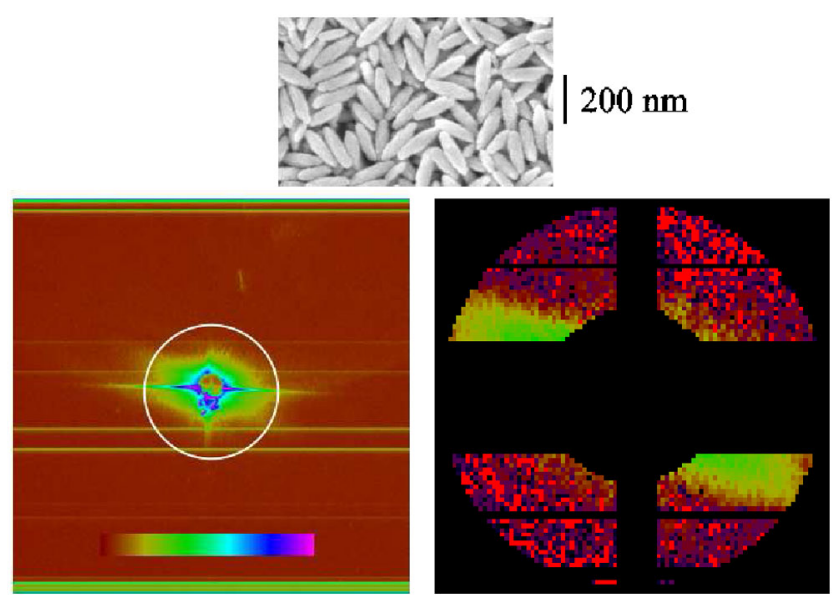

FIG. 1 (color online). Top panel shows an SEM image of the prolate iron oxide nanoparticles (oblate 3D Fourier intensities) used in our experiment. Lower panels show a typical noisy and random diffraction pattern from such a nanoparticle before $(512 \times 512$ array, left $)$ and after processing $(91 \times 91$ array, right): truncated high spatial frequencies (discarded photons counts outside left white circle); binned photon counts; subtracted background; excised nonsignal pixels (redacted). The logarithm of nonzero photon counts are colored according to the inset color bar; negative counts not shown. The mean dynamic range of photon counts in these postprocessed data spans 2 orders of magnitude, subject to fluctuations in fluence.

from a silicon aperture used to shield downstream instruments from beam line scatter. All $5 \times 100$ pulses in a single detector exposure contributed to this scatter whereas only 1 incident pulse creates a useful diffraction pattern if it illuminates a particle. This diffuse background changed gradually as the FEL pulses eroded the edges of the aperture, exacerbating this issue.

To overcome the second challenge, we assumed that the background scatter added incoherently to the diffraction pattern from a pulse illuminating a particle. From 2000 diffraction patterns (data) we identified those that contained coherent scattering (hits), by checking for intensity lobes expected of single prolate nanoparticles (Fig. 1) while excluding anomalous data with scattering from injected debris or those with diffraction patterns from more than one particle. Only nonhits without anomalies were considered background data.

Since the character of the background changed slowly over many data-acquisition cycles, for each hit we compared the results after separately subtracting five background data acquired nearest in time. From these five subtractions we selected the one that gave roughly equal numbers of positive and negative photon counts at higher spatial frequencies, where the particle's signal is presumably negligible [13].

Row defects, scattering from the aperture in the multilayer planar mirror [10] and missing photon counts at the lowest spatial frequencies were always limited to certain pixels of the x-ray sensitive detector. These pixels were excised from all data-their measurements did not constrain intensity reconstruction. $2880\left(M_{\text {pix }}\right)$ usable pixels remained, identical in all hits (nonredacted pixels in Fig. 1, lower right).

Each of the resultant $54\left(M_{\text {data }}\right)$ background-subtracted hits, despite missing information about their orientation $(\Omega)$ and fluence $(\phi)$, are noisy Ewald sphere sections of the particle's true 3D intensities. Our goal is to recover the set of 3D intensities and fluences most statistically compatible with these hits.

Our algorithm for recovering the particle's 3D intensities [4,5] is based on expectation maximization [14], where we iteratively apply a simple rule to increase the compatibility of any model intensities ( $W$, even random ones) with all hits. Consider the simplest case where we are given only one hit. We use a statistical test to determine which Ewald sphere sections in $W$, here on known as tomograms, are most compatible with this hit, then replace those tomograms with said hit, weighted by probabilities taken from the statistical test. This prescription on $W$, which is iterated to a fixed point, determines the most likely orientations of this hit with respect to an increasingly compatible $W$. We can generalize this prescription to many hits by updating the tomograms in consensus with all hits. This Letter extends our algorithm to also determine the fluence distribution of the hits.

The model 3D intensity $W$ has various representations. It can be compactly written as $W(\mathbf{q})$ : the time-integrated scattered intensity at spatial frequency $\mathbf{q}$ when the particle is in some reference orientation. We represented $W(\mathbf{q})$ in our reconstructions as a cubic array of floating-point numbers, indexed by equally spaced samples of $\mathbf{q}$. Detector pixels, labeled by index $i$, measure $M_{\text {pix }}$ point samples $W\left(\mathbf{q}_{i}\right)$.

If we gave the particle some arbitrary orientation $\Omega$ from the reference position, the intensity recorded at detector pixel $i$ is $W\left(\mathbf{R}_{\Omega} \cdot \mathbf{q}_{i}\right)$, where $\mathbf{R}_{\Omega}$ is the orthogonal matrix of this rotation. We approximated the continuous $\Omega$ with a discrete sampling of $M_{\text {rot }}$ points labeled by the index $j$ (sampling discussed in [5]). As a shorthand, we define the intensity at detector pixel $i$, after dividing away the incident pulse fluence, from diffraction off a particle at approximate orientation $j$ as tomogram samples $W_{i j}=$ $W\left(\mathbf{R}_{j} \cdot \mathbf{q}_{i}\right)$.

The statistical test central to our expectationmaximization algorithm assesses the likelihood that measurements on the $i$ th detector pixel of the $k$ th hit $\left(K_{i k}\right)$, with photon fluence $\phi_{k}$, correspond to tomogram samples of our intensity model $W_{i j}$. Intensity fluctuations at each detector pixel of a particular hit due to background subtraction are assumed to be mutually independent. Assuming the noise from background subtraction dominates over Poisson statistics, the likelihood that the $k$ th hit comes from the $j$ th tomogram of $W$ is 


$$
R_{j k}(W, \Omega, \phi) \propto \exp \left(-\frac{\sum_{i}^{M_{\mathrm{pix}}}\left(K_{i k} / \phi_{k}-W_{i j}\right)^{2}}{2 \sigma^{2}}\right) .
$$

The global noise parameter $\sigma$ in Eq. (1) is the only unconstrained parameter in our algorithm. We seek an ideal $\sigma$ that quantifies the true noise in the diffraction data.

Determining the most likely model parameters $(W, \Omega$, $\phi)$ given all hits is unattainable in a single step; hence, we adopt an iterative procedure that we call expansion maximization compression (EMC) to implement the model updates [5].

In the EMC prescription we first expand ( $E$ step) $W(\mathbf{q})$ into the set of $M_{\text {rot }}$ tomograms $W_{i j}$. Although this expansion allows efficient comparisons between hits and model tomograms, it creates redundancy since each intensity sample of the 3D model $W(\mathbf{q})$ is represented in multiple tomograms $W_{i j}$. Such redundancy ameliorates the effects of pixel excisions when populating $W(\mathbf{q})$ with data measurements.

After expanding our model $W$ into tomograms, we maximize ( $M$ step) the data likelihood of each model tomogram $W_{i j} \rightarrow W_{i j}^{\prime}$ independently. Specifically, we determine the new model $\left(W_{i j}^{\prime}, \Omega_{k}^{\prime}, \phi_{k}^{\prime}\right)$ which maximizes Eq. (2), conditional on probabilities of the current model $\left(W_{i j}, \Omega_{k}, \phi_{k}\right)$ :

$$
\operatorname{argmax}_{W^{\prime}, \Omega^{\prime}, \phi^{\prime}} \sum_{k}^{M_{\text {data }}} \sum_{j}^{M_{\text {rot }}} R_{j k}(W, \Omega, \phi) w_{j} \log \left(R_{j k}\left(W^{\prime}, \Omega^{\prime}, \phi^{\prime}\right)\right) .
$$

The numbers $w_{j}$ are weights applied to our rotation group samples that approximate a uniform prior distribution [5]. Despite this uniform prior distribution over the rotation group, we can still detect orientational biases by evaluating Eq. (1) for all hits over the converged $W$ reconstructions.

The requirement that $W_{i j}^{\prime}$ from different orientations are consistent with a single intensity model $W^{\prime}(\mathbf{q})$ is enforced in the final compression ( $C$ step) by averaging interpolated intensity samples in all tomograms (details in [5]), which represent a particular intensity sample in $W^{\prime}(\mathbf{q})$. We also impose Friedel symmetry on $W^{\prime}(\mathbf{q})$ since we are operating in the limit of weak elastic scattering. This compressed and symmetrized model $W^{\prime}(\mathbf{q})$ is now ready for another round of EMC.

We exploited a side effect of EMC's redundant intensity representation to find the ideal noise parameter $\sigma_{\min }$. If $\sigma$ is too small, even though the data likelihood of each updated tomogram is provisionally increased, such tomograms are mutually incompatible, thus diminishing the data likelihood of the compressed updated intensities. We determined $\sigma_{\min }$ knowingly: if $\sigma<\sigma_{\min }$, the log-likelihood of reconstructions, Eq. (2), decline and reconstructions vary dramatically.

EMC updates of the fluence and intensity model have a regrettable degeneracy: if scaling $W^{\prime}(\mathbf{q})$ by a multiplicative constant increases the model's log-likelihood in (2) so will a commensurate scaling in $\phi^{\prime}$. As a consequence, simultaneous EMC updates for $\phi_{k}$ and $W_{i j}$ cannot be decoupled easily. However, if we updated only $\phi_{k}$ or $W_{i j}$, while keeping the other fixed, the net log-likelihood still increases:

$$
\begin{gathered}
W_{i j}^{\prime}=\frac{\sum_{k} R_{j k} w_{j} K_{i k} / \phi_{k}}{\sum_{k} R_{j k} w_{j}}, \\
\phi_{k}^{\prime}=\frac{\sum_{j} R_{j k} w_{j} \sum_{i} K_{i k}^{2}}{\sum_{j} R_{j k} w_{j} \sum_{i} K_{i k} W_{i j}} .
\end{gathered}
$$

The likelihood $R_{j k}$ in the last two equations is evaluated at the current model parameters $(W, \Omega, \phi)$. We imposed $\sum_{j} R_{j k} w_{j}=1$ during each EMC iteration to assert that every hit (index $k$ ) must be found at some orientation (index $j$ ).

We reconstructed 3D Fourier intensities from random starts using EMC with only diffraction data while imposing Friedel symmetry since these are the minimal constraints expected in future cryptotomography experiments. We later evaluated each converged reconstruction (Figs. 2) by fitting them to intensity distributions of ideal ellipsoidal particles $I_{\text {ellip }}$.
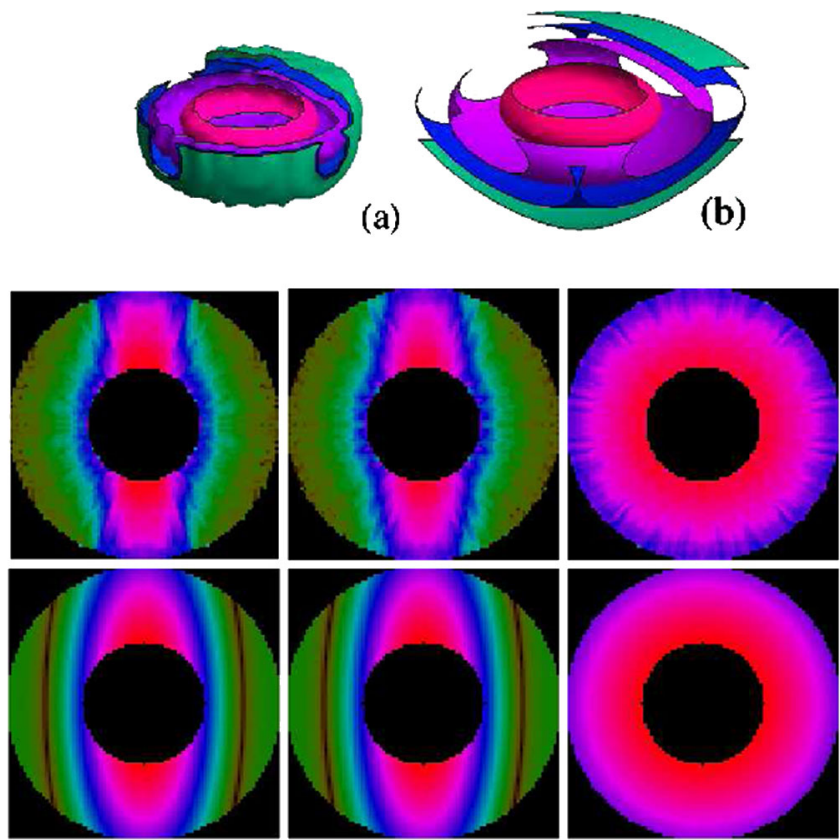

FIG. 2 (color online). Comparing reconstructed intensities to those of an ideal ellipsoidal particle $I_{\text {ellip }}$. (a) Cutaway view of choice 3D isointensity surfaces of a reconstruction which show an oblate intensity distribution and (b) those of $I_{\text {ellip }}$ with the best $R$-factor fit to this reconstruction; (middle row) mutually perpendicular cross sections of this reconstruction; (bottom row) same cross sections of $I_{\text {ellip }}$ in (b). Logarithm of intensities are shown as hues (color bar in Fig. 1). Intensities in reconstructions span 3 orders of magnitude. 


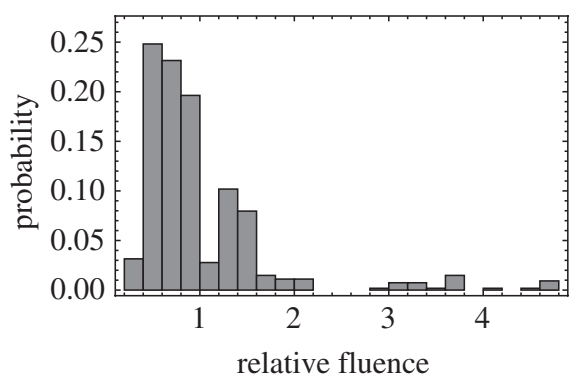

FIG. 3. Probability distribution of reconstructed relative fluence of the hits (expected distributions studied in [12]), a result of fluctuations in pulse fluence and the positions of particles when illuminated.

We began each reconstruction with random intensities $W(\mathbf{q})$, represented by random numbers on a cubic array. Intensities were reconstructed only in the range $q_{\min }<$ $|\mathbf{q}| \leq q_{\max }$ (nonredacted regions in 2D sections of Fig. 2) with $q_{\min } / q_{\max }=20 / 44$ - determined from low spatial frequencies missing-signal region and maximum scattering angle of $5.23^{\circ}$ in processed hits (white circle in Fig. 1, lower left).

We normalized each random initial $W(\mathbf{q})$ to have tomograms that matched the mean power received per hit; initial fluences were set to $\sum_{i} K_{i k}$; we used a $M_{\text {rot }}=3240$ tomographic representation of our model ( $M_{\text {rot }}$ sufficiency discussed in [5]). The noise parameter $\sigma$ was measured in units of $\Delta_{K}$ : the square root of the sum of variances in measured intensities of each pixel [15]. We determined $\sigma_{\min }=0.07 \Delta_{K}$, where reconstructions below this showed diminished likelihood and significant diversity [16].

We could in principle recover the nanoparticle's realspace contrast from the reconstructed 3D intensities $W$ via phase retrieval [7], but there will be pixilation effects (38.3 $\mathrm{nm}$ half-resolution in processed hits). Instead we determined the principal radii of the nanoparticle from our reconstructed intensities using $R$-factor comparisons [17] with those of an ideal ellipsoidal particle $I_{\text {ellip }}(\mathbf{q})$. The particle's principal radii were found to be $30.5 \pm$ $0.8: 30.6 \pm 0.7: 76.1 \pm 1.1 \mathrm{~nm}$ with $R$-factor $0.093 \pm$ $0.006(0.162 \pm 0.003$ when reconstructions were compared to the intensity function expected of the SEM particle measurements) [18]. We expect a larger error in the longer direction of the prolate nanoparticle since it corresponds to the compact direction of its oblate intensities, which was more susceptible to background noise. Figure 3 shows the concomitant fluence distribution we reconstructed.

Figure 4 shows the most likely orientations of the particles corresponding to the 54 hits used in intensity reconstruction [19]. Reconstructed orientational bias in the data could arise from either systematic effects in particle delivery or biases during hit selection. If a nanoparticle's axis of symmetry is colinear with the incident direction of FEL pulses its diffraction pattern will not have identifiable lobes

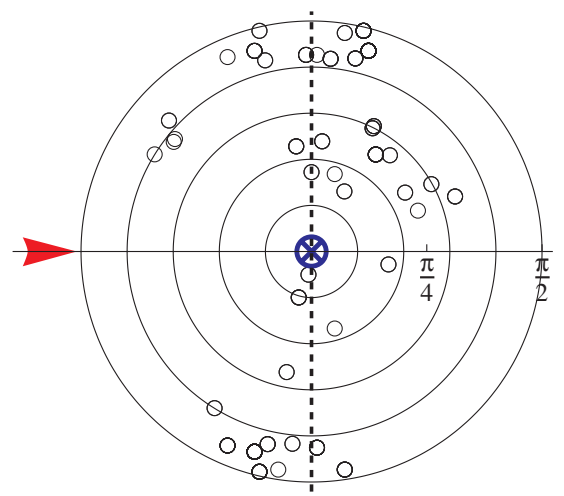

FIG. 4 (color online). We superimpose the most likely orientations of the nanoparticle symmetry axis in the 54 hits found in each of 10 reconstructions in this azimuthal projection (marked by equidistant rings of constant latitude in this top hemisphere). Orientations are inversion symmetric to those in the complementary bottom hemisphere. Many orientations coincide. The vertical dashed line shows the detector plane; FEL pulses traveled with the arrow; nanoparticles were injected into the page (crosshair).

(unlike Fig. 1). Alternatively, data may contain intensity lobes but are obscured by the redacted pixels or background noise. Such data might have been missed during hit selection.

Despite the simplicity of our nanoparticle, we emphasize that reconstructing its 3D intensities, using only diffraction data, is nontrivial primarily because of ambiguities from unmeasured data orientation and fluence. These ambiguities make direct interpretation of the data very challenging. EMC circumvents these ambiguities without prior assumptions about the intensity distribution beyond Friedel symmetry, enforcing only simple statistics, Eq. (1), to determine that the particle was prolate instead of oblate. Subsequent $R$-factor fits of converged EMC reconstructions also gave reasonable particle dimensions, corroborating the effectiveness of EMC on experimental cryptotomography data.

Our work was supported by Helmholtz Association; the PULSE Institute at the SLAC National Accelerator Laboratory by the U.S. Department of Energy, Office of Basic Energy Sciences (M. J. B.); Lawrence Livermore National Laboratory (LLNL) under Contracts No. W7405-Eng-48 and DE-AC52-07NA27344; Laboratory of Directed Research and Development Program of LLNL pertaining to Project No. 05-SI-003; CHESS through NSF and NIH/NIGMS via NSF award DMR- 0225180; Deutsches Elektronen-Synchrotron, DFG Cluster of Excellence at Munich center for Advanced Photonics, Virtual Institute Program of Helmholtz Society, Joachim Herz Stiftung, Max Planck Society and Swedish Research Council. We thank the reviewers for their insightful suggestions and N. D. Loh thanks Yoav Kallus for his invaluable discussions. 
[1] M. J. Bogan et al., Aerosol Sci. Technol. 44, i (2010).

[2] R. Neutze et al., Nature (London) 406, 752 (2000).

[3] H. N. Chapman et al., Nature Phys. 2, 839 (2006).

[4] V. Elser, IEEE Trans. Inf. Theory 55, 4715 (2009).

[5] N.D. Loh and V. Elser, Phys. Rev. E 80, 026705 (2009).

[6] R. Fung et al., Nature Phys. 5, 64 (2009).

[7] H. N. Chapman et al., J. Opt. Soc. Am. A 23, 1179 (2006).

[8] J. Chalupsky et al., Opt. Express 15, 6036 (2007).

[9] M. J. Bogan et al. (unpublished).

[10] S. Bajt et al., Appl. Opt. 47, 1673 (2008).

[11] W. H. Benner et al., J. Aerosol Sci. 39, 917 (2008).

[12] E. L. Saldin et al., The Physics of Free Electron Lasers (Springer-Verlag, Berlin, 1999).

[13] Some potential hits still had strong pure background, due to the mismatched total fluence of potential hits and compared background data, and had to be discarded.

[14] A. P. Dempster et al., J. R. Stat. Soc. Ser. B 39, 1 (1977).

[15] Undesirable rotational averaging occurs at $\sigma=\Delta_{K}$. Such reconstructions resemble $3 \mathrm{D}$ powder diffraction pattern.

[16] Reconstructions using $\sigma_{\min }$ routinely converged to one of two quantitatively distinguishable varieties: log-likelihood of one was about $1 \%$ higher than the other. We rejected the lower-likelihood variety as candidate solutions. Such multiplicity is expected since reconstructions near $\sigma_{\text {min }}$ were only marginally constrained by so few hits (Fig. 4).

[17] $R$ factor between $I_{\text {ellip }}(\mathbf{q})$ and reconstructed $W$ were computed as $\left(\sum_{\mathbf{q}}\left|I_{\text {ellip }}(\mathbf{q})^{1 / 2}-W(\mathbf{q})^{1 / 2}\right|\right) /\left(\sum_{\mathbf{q}} W(\mathbf{q})^{1 / 2}\right)$, where $\left.\left.I_{\text {ellip }}(\mathbf{q}) \propto|(\sin (\pi|\tilde{\mathbf{q}}|)-\pi|\tilde{\mathbf{q}}| \cos (\pi|\tilde{\mathbf{q}}|)) /| \tilde{\mathbf{q}}\right|^{3}\right|^{2} ; \quad|\tilde{\mathbf{q}}|=$ $\sqrt{q_{x}^{2} x_{0}^{2}+q_{y}^{2} y_{0}^{2}+q_{z}^{2} z_{0}^{2}} / q_{\max } ; q_{\min }<|\mathbf{q}| \leq q_{\max } ; x_{0}, y_{0}, z_{0}$ are the principal radii of the nanoparticle. $I_{\text {ellip }}(\mathbf{q})$ and $W$ were normalized to the same total power for $R$-factor comparisons.

[18] We reconstructed ten intensity distributions (e.g., Fig. 2) from random initial intensities. For each reconstruction we found the principal radii that gave the best $R$-factor fit to an ellipsoidal intensity $I_{\text {ellip }}(\mathbf{q})$; the quoted radii are their averages.

[19] EMC found the particle's axial symmetry without imposition. For each hit, EMC automatically assigned nearly equal probability to orientations related by this symmetry, hence mitigating data-scarcity effects expected from limited hits, pixel excision and, orientation biases. 